\title{
Una espina en un pajar: espina de pescado alojada en la tiroides
}

\author{
A fish bone in a haystack: fish bone lodged in thyroid \\ Jean Pulido ${ }^{1,2} \mathbb{D}$, Alejandro Sánchez $2,3 \mathbb{D}$, Andrey Moreno ${ }^{2,3} \mathbb{D}$ \\ Departamento de Medicina General, Universidad El Bosque, Bogotá, D.C., Colombia \\ Departamento de Cirugía General, Hospital Universitario de La Samaritana, Bogotá, D.C., Colombia \\ Departamento de Cabeza y Cuello, Hospital Universitario de La Samaritana, Bogotá, D.C., Colombia
}

\section{Resumen}

Introducción. La ingestión accidental de cuerpos extraños como espinas de pescado es muy poco frecuente; en ocasiones, pueden alojarse en el esófago y es posible su migración extraluminal. Este accidente se presenta principalmente en países asiáticos por el gran consumo de pescado. La migración extraluminal de una espina de pescado desde el esófago a la glándula tiroides es aún más infrecuente, con pocos reportes en la literatura.

Objetivo. Presentar nuestra experiencia en el diagnóstico y manejo quirúrgico de un paciente con un cuerpo extraño (espina de pescado) alojado en la glándula tiroides y, asimismo, hacer una revisión de la literatura científica.

Caso clínico. Se presenta una paciente de 53 años, sin antecedentes de importancia y con un cuadro clínico de tres meses de evolución consistente en odinofagia posterior a la ingestión accidental de una espina de pescado. En la ecografía practicada en otra institución se observó un cuerpo extraño alojado en el lóbulo tiroideo izquierdo en relación con la musculatura del esófago. En la tomografía de cabeza y cuello se apreciaba una imagen hiperdensa lineal de 2,6 cm de longitud en la misma localización. En la exploración quirúrgica se encontró tejido fibrótico en la región posterior del lóbulo tiroideo izquierdo y se procedió a practicar una tiroidectomía subtotal izquierda. La evolución posoperatoria fue adecuada.

Conclusiones. La migración extraluminal de cuerpos extraños en el tubo digestivo es factible, sobre todo la de las espinas de pescado por su morfología. La tomografía es la herramienta diagnóstica más confiable para detectar cuerpos extraños alojados en la glándula tiroides. Es necesario conocer las posibles complicaciones y, asimismo, optimizar las posibles intervenciones, pues de eso depende el pronóstico clínico del paciente.

Palabras clave: cuerpos extraños; migración de cuerpo extraño; absceso; glándula tiroides; tiroidectomía; cirugía.

Fecha de recibido: 2/10/2019 - Fecha de aceptación: 18/11/2019

Correspondencia: Jean Pulido Segura, Carrera 20 \# 134 - 38, Bogotá, D.C., Colombia. Teléfono: (57) (313) 330-9984

Correo electrónico: japs1493@gmail.com

Citar como: Pulido J, Sánchez A, Moreno A. Una espina en un pajar: espina de pescado alojada en la tiroides. Rev Colomb Cir. 2020;35:491-7. https://doi.org/10.30944/20117582.484

Este es un artículo de acceso abierto bajo una Licencia Creative Commons - BY-NC-ND https://creativecommons.org/licenses/by-ncnd/4.0/deed.es 


\section{Summary}

Introduction: Accidental ingestion of foreign bodies such as fish bones is very rare; sometimes they may lodge in the esophagus, and extraluminal migration is possible. This accident occurs mainly in Asian countries due to the large fish consumption. Extraluminal migration of a fish bone from the esophagus to the thyroid gland is even more infrequent, with few reports in the literature.

Objective: To present our experience in the diagnosis and surgical management of a patient with a foreign body (fish bone) lodged in the thyroid gland and to review the scientific literature.

Clinical Case: A 53-year-old patient is presented, without a significant history and with a clinical picture of three months of evolution consisting of odynophagia after accidental ingestion of a fish bone. In the ultrasound performed at another institution, a foreign body was observed lodged in the left thyroid lobe in relation to the musculature of the esophagus. Head and neck tomography showed a linear $2.6 \mathrm{~cm}$ long hyperdense image at the same location. On surgical exploration, fibrotic tissue was found in the posterior region of the left thyroid lobe and a left subtotal thyroidectomy was performed. Postoperative evolution was adequate.

Conclusions: Extraluminal migration of foreign bodies in the digestive tract is feasible, especially that of fish bones due to their morphology. Tomography is the most reliable diagnostic tool to detect foreign bodies lodged in the thyroid gland. It is necessary to know the possible complications and to optimize the possible interventions, since the clinical prognosis of the patient depends on it.

Key words: foreign bodies; foreign-body migration; abscess; thyroid gland; thyroidectomy; surgery.

\section{Introducción}

La ingestión accidental de cuerpos extraños, como espinas de pescado, es muy poco frecuente; en ocasiones, pueden alojarse en el esófago y es posible su migración extraluminal. Este accidente se presenta principalmente en los países asiáticos por el gran consumo de pescado. Más rara aún es la migración extraluminal, la cual, a pesar de pasar desapercibida en algunos casos, puede tener consecuencias que afecten la salud, como la formación de abscesos o la localización en otras estructuras anatómicas, por ejemplo, en la glándula tiroides.

La diversidad de la sintomatología, de los métodos diagnósticos y de los tratamientos disponibles, nos motiva para informar nuestra experiencia en el manejo quirúrgico de una paciente con ingestión accidental de una espina de pescado que migró desde el esófago hasta alojarse en el lóbulo tiroideo izquierdo, sin dañar ninguna otra estructura en su trayecto, y a hacer una revisión de la literatura científica. A pesar de ser un hallazgo infrecuente, es un accidente potencialmente mortal que debe reconocerse y tratarse de manera oportuna para disminuir el riesgo de complicaciones.

\section{Caso clínico}

Se presenta el caso de una paciente de 53 años de edad, sin antecedentes de importancia, con un cuadro clínico de tres meses de evolución de odinofagia posterior a la ingestión accidental de una espina de pescado. Ingresó con un reporte de una ecografía extrainstitucional que había demostrado un cuerpo extraño alojado en el lóbulo tiroideo izquierdo en relación con la musculatura del esófago. Se le tomó una tomografía computarizada (TC) de cabeza y cuello que mostraba una imagen hiperdensa lineal de 2,6 cm de longitud en la misma localización.

Dado el tiempo de evolución y el compromiso extraluminal del cuerpo extraño, se procedió a la exploración quirúrgica y se encontró tejido fibrótico en la región posterior del lóbulo tiroideo izquierdo, como reacción a un cuerpo extraño espinoso que perforó la pared antero-lateral del 
esófago cervical y comprometía la adventicia, por lo cual se practicó una tiroidectomía subtotal izquierda.

Aunque la evolución durante el periodo posoperatorio inmediato fue adecuada, a los diez días del egreso la paciente volvió a consultar por infección superficial del sitio operatorio. La ecografía de tejidos blandos no fue concluyente y se solicitó una TC con contraste de cuello, en la cual se evidenció una colección con posible sobreinfección, con un volumen de $3,7 \mathrm{ml}(30 \mathrm{x}$ $9 \mathrm{~mm}$ en el plano axial y $27 \mathrm{~mm}$ en el coronal), y adenomegalias de tipo reactivo. Se drenó la colección y su cultivo fue negativo. La evolución de la paciente fue satisfactoria.

\section{Revisión de la literatura}

\section{Epidemiología}

La ingestión accidental de cuerpos extraños, especialmente de espinas de pescado, es un problema común que afecta especialmente a la población asiática por el gran consumo de estos alimentos, aunque también suele ser un problema común en población pediátrica, adultos con enfermedades psiquiátricas, alcohólicos y en pacientes sin dientes ${ }^{\mathrm{I}-4}$.

A pesar de que del 80 al $90 \%$ de los cuerpos extraños pasan sin causar ningún daño en el tubo digestivo ${ }^{2}$, algunos, como las espinas de pescado, pueden incrustarse ${ }^{3}$. Esto requiere manejo médico conservador hasta en el $20 \%$ de los casos o intervención quirúrgica en el I \%, esta última relacionada con la incidencia de la migración extraluminal de las espinas de pescado ${ }^{2,5}$.

El principal sitio de perforación de las espinas de pescado es el esófago ${ }^{4}$, seguido por las amígdalas palatinas, la base de la lengua, la vallécula epiglótica, los senos piriformes y el esófago ${ }^{ }$. Sin embargo, como en el presente caso, puede presentarse migración extraluminal de la espina de pescado. Aunque es poco frecuente, se ha descrito en la literatura científica, y por primera vez en 1949 por Jemerin y Aronoff. Estos autores informaron sobre un cuerpo extraño que migró de la orofaringe a la glándula tiroides ${ }^{15,6}$. Esta localización final es aún más extraña debido a las estructuras anatómicas que se interponen y se cuenta con pocos casos reportados 7 . Como consecuencia, es posible que se formen abscesos tiroideos, más comúnmente en la población pediátrica, y su aparición está directamente relacionada con el tiempo que el cuerpo extraño permanezca alojado en el esófago. Los microorganismos más comúnmente aislados son estafilococos y diversas especies de estreptococos, aunque los cultivos suelen ser polimicrobianos 5,8 .

\section{Fisiopatología}

El mecanismo exacto de la migración se desconoce, pero se cree que se relaciona con el peristaltismo del esófago, el movimiento del cuello y las características físicas de las espinas de pescado, ya que su estructura es fina, lineal y filosa (figura I), lo cual les permiten atravesar fácilmente la mucosa con la fuerza que los músculos alrededor ejercen sobre ellas e, incluso, llegan a migrar fuera de la luz esofágica en horas, lo cual aumenta el riesgo para el paciente ${ }^{\mathrm{I}, 7}$.

\section{Sintomatología}

La sintomatología de esta entidad es variada y puede presentarse de manera aguda o crónica. Los síntomas más frecuentes son dolor de garganta y del tórax (88\%), sensación de cuerpo extraño (54\%), odinofagia y disfagia (6-67\%), vómito (42\%), fiebre (8-20 \%), hemoptisis (6\%)

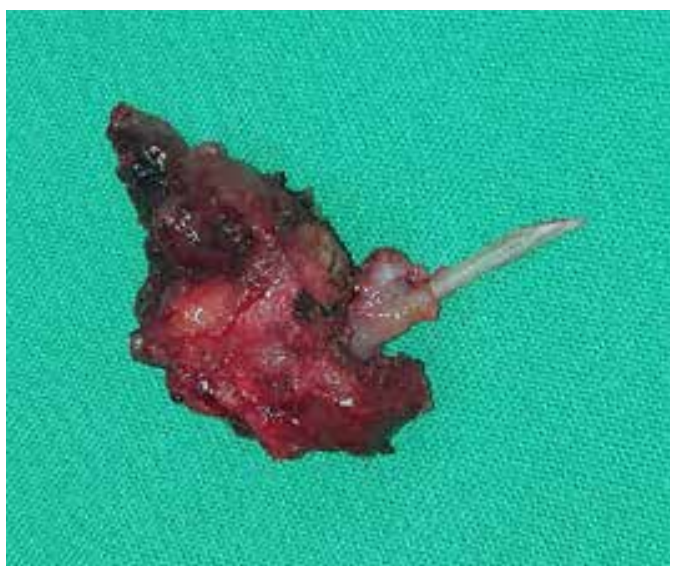

Figura 1. Pieza quirúrgica con espina de pescado visible 
y sialorrea. En el presente caso, la odinofagia fue el único síntoma manifestado ${ }^{2-4,6}$.

El tiempo de aparición de los síntomas también es muy variable. En ocasiones, el cuerpo extraño ha estado incrustado por mucho tiempo ${ }^{\mathrm{I}}$ y la instauración de los síntomas puede tardar semanas ${ }^{6}$, como en esta paciente, que lo había ingerido, aproximadamente, 3 meses antes. Los cuerpos extraños incrustado por más de 24 horas tienen I4,I veces más posibilidades de generar complicaciones ${ }^{\mathrm{I}}$. Kenichi, et al., reportaron un paciente que retuvo una espina de pescado durante 9 meses y mencionan otros con cuerpos extraños en el músculo esternocleidomastoideo, incluso, hasta por dos años ${ }^{\mathrm{I}}$.

Las complicaciones por un cuerpo extraño son infrecuentes, pero su morbilidad y mortalidad son importantes ${ }^{2,47}$. Antes de la era de los antibióticos, la mortalidad llegaba incluso al $45 \%$. Hoy en día, se calcula una mortalidad de o a $0,73 \%{ }^{1,5} \mathrm{y}$, en los Estados Unidos, llega a I.500 muertes por año, aproximadamente ${ }^{2}$.

La perforación esofágica se puede evidenciar por periesofagitis, absceso periesofágico, mediastinitis o complicaciones vasculares, como fístula aorto-esofágica, fístula esofágica innominada, fístula esofágica subclavia y ruptura de carótida ${ }^{5,9}$. También pueden existir procesos locales supurativos, absceso retrofaríngeo, absceso tiroideo o absceso profundo de cuello ${ }^{\mathrm{I}}$. La paciente que presentamos solo tuvo perforación a la altura de la glándula tiroides y un absceso secundario.

\section{Diagnóstico}

Una historia clínica detallada y un examen físico exhaustivo pueden aportar los primeros indicios para determinar qué herramienta diagnóstica utilizar ${ }^{5,9,10}$. Cuanto más tiempo dure el cuerpo extraño en el esófago, es mayor el riesgo de perforación, por lo cual, el diagnóstico rápido es esencial ${ }^{1,2,4, \mathrm{II}}$.

Ante la sospecha de un cuerpo extraño alojado en las vías digestivas, el examen inicial suele ser una radiografía simple de cuello, por su disponibilidad, aunque esta solo alcanza el $80 \%$ de sensibilidad. En combinación con la TC, alcanza el $100 \%{ }^{6,9} \mathrm{y}$, además, permite guiar el enfoque hacia la eliminación de la espina de pescado. Con estos dos estudios, se puede demostrar la ruta migratoria y se detecta un buen número de cuerpos extraños delgados, pequeños y mínimamente calcificados 5,7,10,II (figura 2).

La endoscopia de las vías digestivas altas ha sido utilizada también como un método diagnóstico. Lai, et al., incluyeron en su estudio I.338 pacientes con diagnóstico de sospecha de ingestión de cuerpo extraño; a I.028 se les practicó endoscopia de las vías digestivas altas, pero tan solo en el $39 \%$ se demostró la existencia de un cuerpo extraño y, de este porcentaje, en el 92,3\% fue removido con éxito ${ }^{2}$.

La falta de detección de un cuerpo extraño por endoscopía no descarta su presencia ${ }^{4}$, Por ejemplo, Benítez, et al., reportaron el caso de una paciente de 54 años de edad con cuadro de dolor torácico y epigástrico, con un reporte negativo de la endoscopia de las vías digestivas altas, en

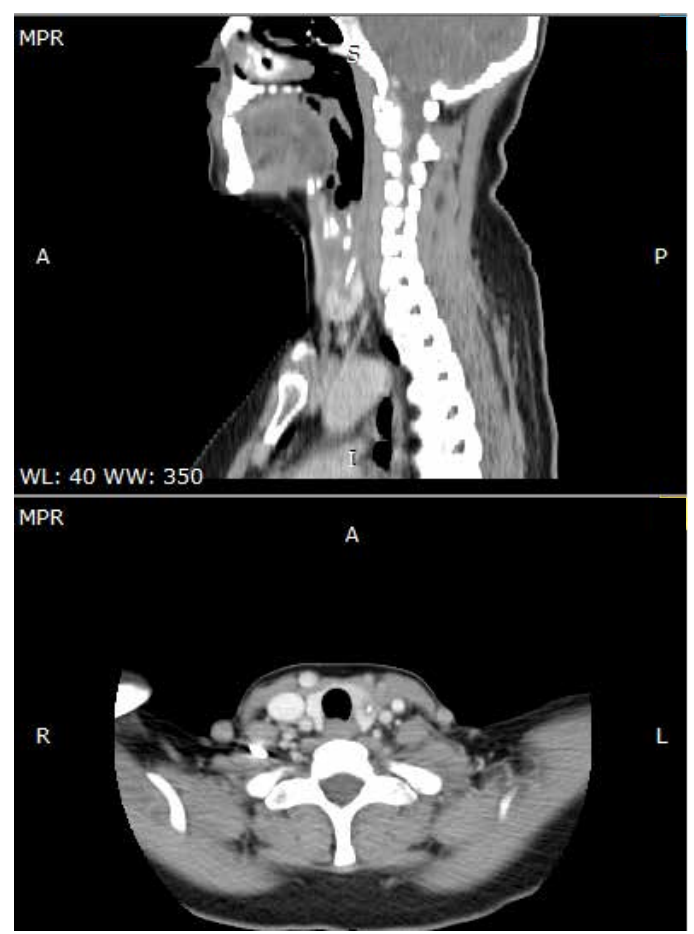

Figura 2. Tomografía computarizada de cuello en la que se visualiza el cuerpo extraño. 
quien, mediante angiotomografía, se diagnosticó ruptura de la aorta torácica asociada a ruptura de las paredes esofágicas luego de la ingestión de una espina de pescado que, en un principio, solo se manifestó con sensación de cuerpo extraño ${ }^{12}$.

Los otorrinolaringólogos ocasionalmente usan el laringofibroscopio flexible para descubrir lesiones en la mucosa del esófago faríngeo, pero, en muchos casos, este puede estar cubierto por tejido cicatricial; además, su utilización es complicada y el retraso en los hallazgos puede llevar a complicaciones innecesarias ${ }^{1,5}$.
La ecografía de cuello es una herramienta de gran utilidad que permite evidenciar los cuerpos extraños por la sombra acústica que producen ${ }^{8}$ $y$, en algunos casos, como en el presente, se usa como ayuda diagnóstica inicial.

Algunos autores recomiendan el uso de la ultrasonografía intraoperatoria por su disponibilidad y costo-efectividad ${ }^{1,5}$.

\section{Tratamiento}

Llama la atención el algoritmo de manejo propuesto por Lai et al., (figura 3), como resultado

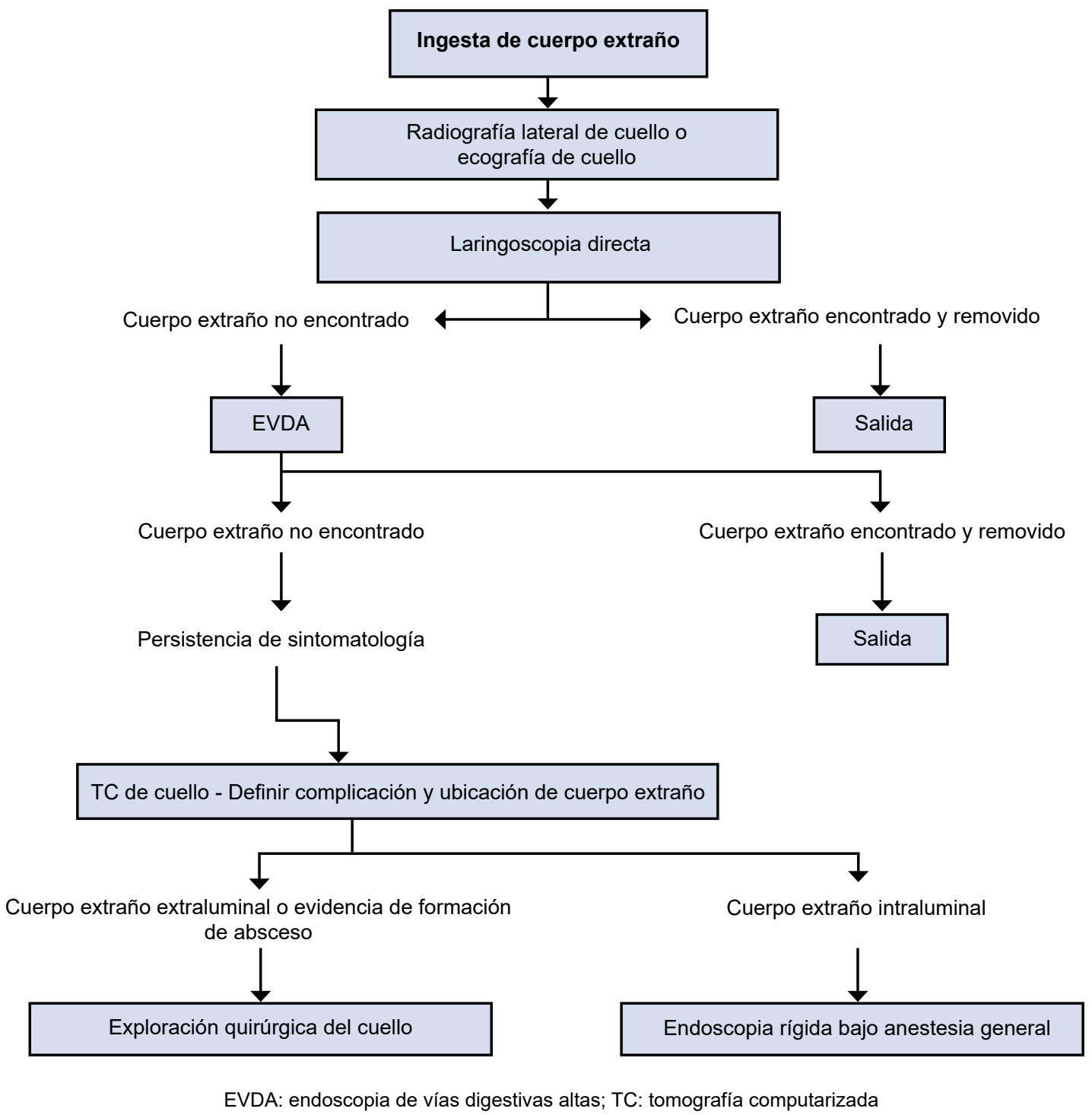

Figura 3. Modificado con permiso del algoritmo de manejo propuesto por Lai, et al., 2,6. 
de un estudio de I.338 pacientes con diagnóstico de sospecha de ingestión de cuerpo extraño ${ }^{2}, y$ agregamos la posibilidad de la ecografía como método diagnóstico inicial en conjunto con la radiografía. Por otra parte, proponemos la TC para detectar posibles complicaciones, la ubicación exacta y el trayecto migratorio, con el propósito de lograr un adecuado abordaje en la exploración cervical.

Suele preferirse la exploración quirúrgica del cuello cuando los cuerpos extraños migran fuera de las vías digestivas y, asimismo, cuando se forman abscesos o la endoscopia de las vías digestivas altas falla, como sucedió en el presente caso, que la paciente fue sometida a exploración quirúrgica tras un resultado endoscópico negativo.

La técnica abierta suele ser la principal elección, pues permite llegar fácilmente al sitio lesionado, así como evitar lesiones de otras estructuras adyacentes por la visualización directa ${ }^{1,2,5,8,10}$. Entre otras, estas fueron las razones por las que se decidió la cirugía abierta en esta paciente (figura 4).

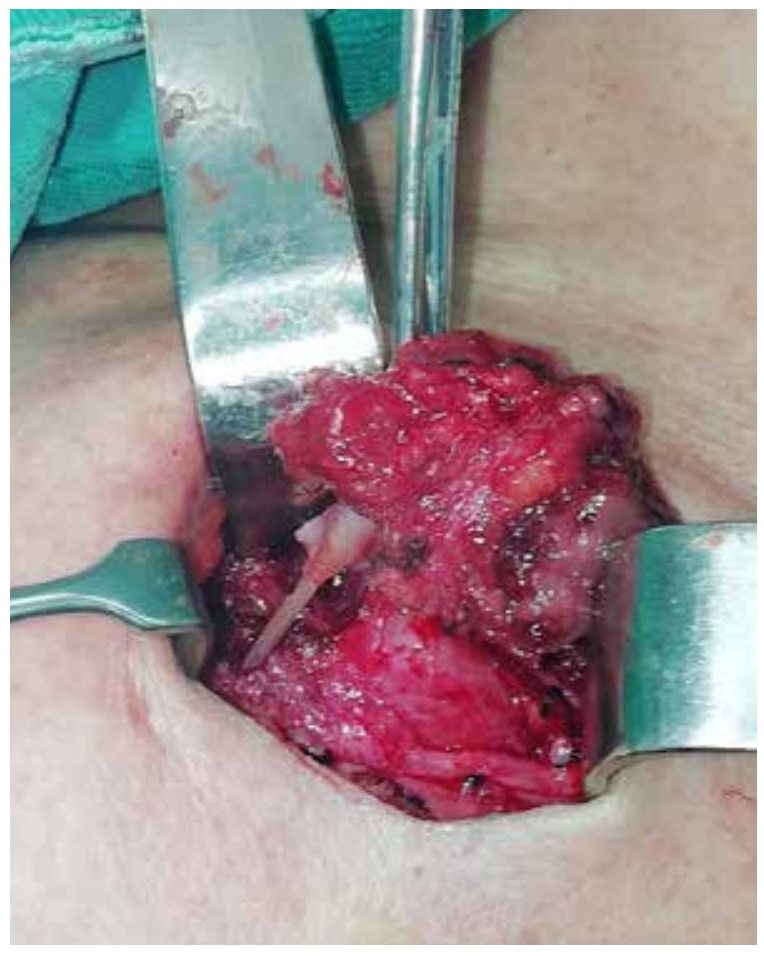

Figura 4. Exploración cervical abierta en la que se observa la espina de pescado alojada en la glándula tiroides.
Asimismo, se debe aprovechar la intervención quirúrgica para tomar una muestra y hacer un cultivo, para orientar la antibioticoterapia 5 .

A pesar de ser el abordaje quirúrgico abierto la principal opción de manejo, se debe tener en cuenta el nivel del tubo digestivo comprometido pues, como lo demostraron Madagán, et al., hoy en día existen opciones mínimamente invasivas que pueden ayudar en el tratamiento de esta entidad ${ }^{13}$.

\section{Conclusiones}

La migración extraluminal de cuerpos extraños fuera de las vías digestivas es factible, sobre todo el de las espinas de pescado debido a su morfología. La TC es la herramienta diagnóstica más confiable para detectar cuerpos extraños alojados en la glándula tiroides. El abordaje quirúrgico abierto es la mejor opción, pues permite la visualización directa de las estructuras comprometidas y, asimismo, disminuye el riesgo de lesiones de las estructuras adyacentes.

\section{Cumplimiento de normas éticas}

Consentimiento informado. Este estudio se basó en una revisión bibliográfica y en el análisis retrospectivo de la historia clínica. Según la Resolución 008430 de 1993 del Ministerio de Salud de Colombia, se consideró que la investigación no tenía riesgo para la paciente, por lo tanto, no se diligenció un consentimiento informado.

Conflicto de interés. Ninguno reportado por los autores.

Fuentes de financiación. Los autores declaramos no contar con financiamiento de terceros.

\section{Referencias}

I. Watanabe K, Amano M, Nakanome A, Saito D, Hashimoto $\mathrm{S}$. The prolonged presence of a fish bone in the neck. Tohoku J Exp Med. 2012;227:49-52. https://doi.org/IO.I620/tjem.227.49

2. Lai ATY, Chow TL, Lee DTY, Kwok SPY. Risk factors predicting the development of complications after foreign body ingestion. Br J Surg. 2003;90:I53I-5. https://doi.org/IO.IO02/bjs.4356 
3. Wu E, Huang L, Zhou Y, Zhu X. Migratory fish bone in the thyroid gland: Case report and literature review. Case Rep Med. 2018:7345723

https://doi.org/IO.II55/2018/7345723

4. García-Suárez L, González-Sánchez S, Vivanco-Allende A, Anes-González G. Absceso tiroideo secundario a perforación esofágica por espina de pescado. An Pediatr (Barc). 2019;92:I74-5. https://doi.org/IO.IOI6/j.anpedi.2019.0I.006

5. Chen CY, Peng JP. Esophageal fish bone migration induced thyroid abscess: Case report and review of the literature. Am J Otolaryngol. 20II;32:253-5. https://doi.org/Io.IoI6/j.amjoto.2010.02.006

6. Hohman MH, Harsha WJ, Peterson KL. Migration of ingested foreign bodies into the thyroid gland: Literature review and case report. Ann Otol Rhinol Laryngol. 20IO;II9:93-8. https://doi.org/IO.II77/00034894IOII900205

7. Chen H, Ruan L, Zhou S, Wang S. The utility of repeated computed tomography to track a foreign body penetrating the esophagus to the level of the thyroid gland. Oral Radiol. 2014;30:196-202. https://doi.org/IO.IOO7/sII282-0I3-OI56-y

8. Ohbuchi T, Tabata T, Nguyen K, Ohkubo J, Katoh A, Suzuki $\mathrm{H}$. Thyroid gland cutaneous fistula secondary to a migratory fish bone: A case report. J Med Case Rep. 20I2;6:I4O. https://doi.org/IO.II86/I752-I947-6-I4O
9. Liu CH, Huang SC, Lin WS, Lin CS. Unrecognized esophageal fish bone impaction with delayed presentation of acute spastic central chest pain. Medicine (Baltimore). 2019;98:eI4627.

https://doi.org/IO.I097/MD.00oooooooool4627

Io. Johari HH, Khaw B-L, Yusof Z, Irfan M. Migrating fish bone piercing the common carotid artery, thyroid gland and causing deep neck abscess. World J Clin Cases. 2016;4:375-9.

https://doi.org/IO.I2998/wjcc.v4.iII.375

II. Petrarolha SMP, Dedivitis RA, Perruccio FG, Quirino IdA. Esophagus foreign body in the thyroid gland. Braz J Otorhinolaryngol. 20I7;SI808-8694(I7)30149-0. https://doi.org/IO.IOI6/j.bjorl.20I7.08.008

I2. Benítez E, Alfonso M, Lesmes M, Barón V, Hernández O, Oliveros G. Perforación aorto-esofágica, diagnóstico clínico e imagenológico: reporte de dos casos clínicos con manejo endovascular. Rev Colomb Cir. 2019;34:190-8. https://doi.org/I0.30944/20117582.II4

I3. Magadán C, Fidalgo M, ToledoE, Olmos JM, Terán A, Rodríguez-Sanjuán JC, etal. Perforación de colon sigmoides por espina de pescado: tratamiento combinado laparoscópico y endoscópico. Rev Colomb Cir. 2018;33:433-7. https://doi.org/I0.30944/20117582.9I 